\title{
Scalable approach in Greedy Perimeter Stateless Routing
}

\author{
Neha Chachra \\ Punjab Technical University \\ Kapurthhala, Jalandhar-India
}

\author{
Jogishwar Singh Sohal \\ Punjab Technical University \\ Kapurthhala, Jalandhar-India
}

\begin{abstract}
Greedy perimeter stateless routing (GPSR), a scalable routing protocol for wireless sensor networks (WSNs) that use the randomized positioning of routers in different configurations and algorithms are used to make packet delivery decisions through nodes. As the number of destinations increases this protocol scales better in per-router state than shortest-path and ad-hoc routing protocols. Because of scalable topology property in GPSR, it uses local topology (e.g. star, ring) to find out new and correct routes quickly as per demand. In this paper, we describe the GPSR protocol and optimization of mobile wireless networks to compare its performance based on changes in topology. Our simulation elaborates GPSR's scalability on densely deployed wireless networks based on route adaptability.
\end{abstract}

\section{Keywords}

GPSR, WSN, Scalability

\section{INTRODUCTION}

In wireless networks because of entirely dense connected wireless stations, data gathering information may require multiple hoping, because of availability of finite radio ranges. Although community of networking has observed, demonstrated, implemented a number of routing schemes for ad hoc networks [1], [2]. The use of distance vector (DV), link state (LS) and path vector algorithms [3], [4] are used for scalable topology in wireless networks for efficient routing related to a given application. DV's Bellman-Ford approach makes this global data information transitively; in this each router includes its distance from all network destinations in each of its periodic beacons after a particular interval of time. LS's Digkstra algorithm directly gives information of the change in any link status to every router in the network. Small inaccuracies found in both DV and LS can cause routing loops or disconnection [5]. When the topology is in constant flux, as under mobility, LS generates torrents of link status change messages, and DV either suffers from out-of-date state or generates torrents of triggered updates. As the number of destinations increases hierarchy plays a very vital role to scale the routing parameters. The hierarchy is based on rarely changing administration with topological boundaries. So it is not easily approachable for freely moving ad hoc wireless networks, to have scalable topology characteristic.

Another approach related to scalable topology is Caching. Dynamic Source Routing (DSR) [6], Ad-hoc on demand distance vector routing (AODV) [7] and Zone routing protocol (ZRP) [8] all constantly inform about the current topology information in an on-demand scenario as recommended by their packet forwarding load and to cache it accurately. If the topology becomes out-of-date then current topological updates are required.

The main task of caching is to reduce overloading of packets in two ways:- a. Without requirements; any topological change does not acceptable.

b. Decrease the number of hops between the routes.

We propose the efficient use of area under geography to get scalability in this wireless routing protocol known as Scalable Greedy Perimeter Stateless Routing. Scalability enhances the numbers of nodes and mobility rate.

The factors under scalability are:-
a. Numbers of packets send.
b. Success delivery ratio.
c. Memory storage at each node.

\section{GPSR}

In GPSR, routers are assumed to be stateless for propagation of topology information where each node's need is only to know its neighbor's positions. The usefulness of routing is attained through self-describing nature of positions of nodes. By knowing the position of a packet's destination and positions of the candidate next hops, are sufficient to make correct decision regarding routing forwarding. The wireless routers have complete information about their own positions through GPS services in outdoors, inertial sensors on vehicles, range finding using radar and ultrasonic chirps. The IEEE 802.11 wireless network MAC [9], [10] sends link based acknowledgements for uni-cast packets, with the bidirectional links in an 802.11.

\section{GRAPHICAL APPROACH}

Under the graphical approach the no-crossing demand of routing in GPSR is deal with the planar graphs. The graphs in which no two edges are crossing are known as planarized graphs. Graph is a composition of nodes with specified radio range, where all radios have identical, circular range $r$; and each node is treated as vertex with edge formation (m:n) exists between nodes $m$ and $n$. The condition under radio range ' $r$ ' is $d(m, n)<=r$.

\subsection{Types of Graphs}

a. Relative neighborhood graph (RNG)

b. Gabrial Graph (GG)

Relative neighborhood graph (RNG): Removing edges from the graph does not mean to disconnect the graph, but to partition the network shown in fig. 1 .

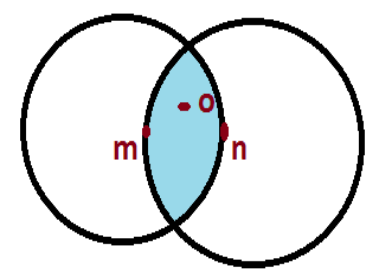

Fig.1 RNG (Relative neighborhood graph) 
RNG Algorithm:

An edge (m:n) exsists between vertices $m$ and $n$ with the distance between them $d(m: n)$, is less

than or equal to the distance between every other vertex 'o' and being of $\mathrm{m}$ and $\mathrm{n}$ farther from ' $\mathrm{o}$ '.

In equational form:-

$\mathrm{m}, \mathrm{n}: \mathrm{d}(\mathrm{m}: \mathrm{n})<=$ [d (m:o); d(n:o)]

The shaded region is the intersection area of two networks shown in fig. 1 .

for all $\mathrm{n} € \mathrm{~N}$ do; where $\mathrm{N}$ is the total number of neighbors

for all o $€ \mathrm{~N}$ do

if $\mathrm{o}==\mathrm{n}$ then

continue

else if $d(m: n)>\max [d(m: o) ; d(n: o)]$ then

eliminate edge (m:n)

break

end if

end for

end

Gabrial Graph (GG):

In this graph the mid-point of edge m-n is to be determined shown in fig.2.

shown in fig. 2 .

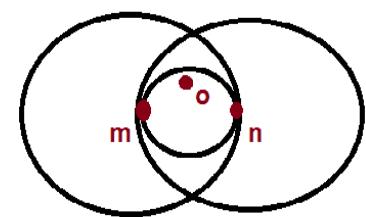

Fig.2. GG (Gabrial graph)

An edge $(m, n)$ exists between vertices $m$ and $n$ if no other vertex 'o' present within the circle whose is diameter equals to the diameter of present edge $(\mathrm{m}, \mathrm{n})$.

In equatorial form:

$\mathrm{u}, \mathrm{v}: d 2(\mathrm{~m}, \mathrm{n})<[d 2(\mathrm{~m}, \mathrm{o})+d 2(\mathrm{n}, \mathrm{o})]$

GG Algorithm:

$\mathrm{M}=$ mid- point of $\mathrm{mn}$

for all $\mathrm{m} € \mathrm{~N}$ do

for all o $€ \mathrm{~N}$ do

if $n==o$ then

continue

else if

$\mathrm{d}(\mathrm{M}, \mathrm{o})<\mathrm{d}(\mathrm{M}, \mathrm{m})$ then

eliminate

edge $(m, n)$

break

end if

end for

end for

Removing edged in the GG cannot disconnect a connected graph unit, as same as in RNG. The time taken by the algorithms RNG and GG at each node is O (deg2), where deg is the node's degree in the complete radio graph. The RNG is a subset of GG. GG can work under smaller area with less complexity as compared to RNG.

\section{SCALABLE APPROACH}

SGPSR (Scalable Greedy Perimeter Stateless Routing) has vigorously implemented on IEEE 802.11 network. To make GPSR vigorous on a mobile IEEE 802.11 network, we made the considerable choices adopted are:

\subsection{Queue Interfacing}

In IEEE interface the packets are sent by queue after getting link level

acknowledgements from the receiver. In addition to this if retransmit retry failure, there is a traverse of queue of packets and removal of all packets related to the failed transmission. Then there is a procedure to re-forwarding of packets in next hoop.

\subsection{MAC-failure}

Failure exists in 802.11 MAC layer when packets exceeds its maximum number of retransmission limitation. It happens under out of range radio range. It gives the information to the protocol about expiration of the neighbor's time exceed interval.

\subsection{Efficient use of Network interface}

It considers the availability of packets with the beaconing interval within specified radio range in the network. The list is updated continuously with the present traffic load related to their neighborhood.

\subsection{Planarized graphs}

RNG and GG graph theory is used to get current information of positioning of nodes information. The loss of packets is distinguished by beacon interval. MAC is used to indicate failure packet transmission. The need is to update planarized graphs depends upon the present load consideration.

\section{IMPLEMENTATION}

To attain the design goals for SGPSR, the algorithm is specified for a number of mobile network topologies. Topological scalability and mobility are main considerations to be adopted in this scenario.

\subsection{Simulation Environment}

The ns-2.34 wireless simulation tool is used to simulate nodes stirring in an unobstructed environment. Node chooses the destination in a uniform plane in random motion in the given region. The main focus of nodes is to attain the destination with the chosen velocity and a pause time is acceptable before the repetition cycle repeats.

\subsection{Program variables selected by the user are \\ - Nodes radio range \\ - Algorithms \\ - Random movement range \\ - $\quad$ Network size \\ - Network density \\ - $\quad$ Beacon periods \\ - $\quad$ Type of Network (GPSR, Greedy)}

\subsection{Simulation parameters}

In the simulation, the network is for $30,50,100$ nodes with 802.11 radios, with the nominal 150

meter range. The nodes are placed uniformly in a rectangular region at random motion. All nodes have velocity of $15 \mathrm{~m} / \mathrm{s}$. The pause times during simulation are $0,5,10,15,20$ seconds as shown in Fig 3. The CBR is calculated at 30 bit rate traffic load. Each CBR traffic rate is $3 \mathrm{Kbps}$ and uses 64-byte packets to be sent 


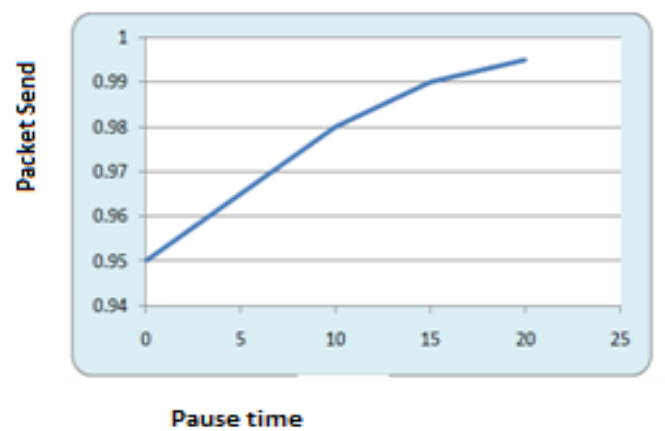

Fig.3 Packet ratio send

\section{ALGORITHMS COMPARISON}

The mobile network performance depends upon the transmission range of packets related to a particular algorithm. Under the greedy forwarding the algorithm that can be used by the user are DSDV, TORA. The GPSR capability to send packets under the transmission range is more than RNG and Greedy with the successful rate of transmission of packets with lee retransmissions as shown in fig. 4 .

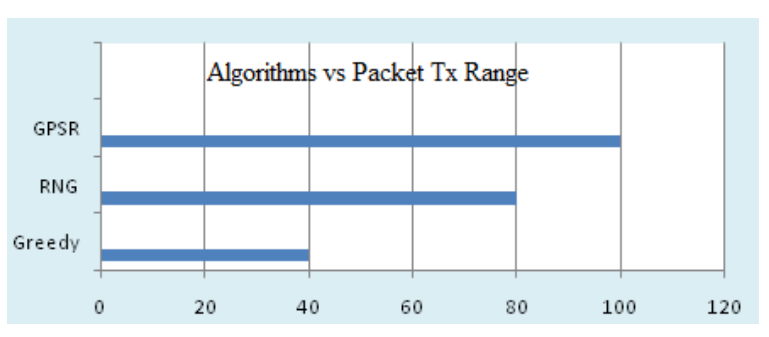

Fig.4. Algorithmic Comparison

Topological Comparison on GPSR: The GPSR protocol depends upon the scalable topology consideration. Fig.5 shows the differences between Static Ring, Star, Ring and Static star with respect to the packets transmission range. Static ring is the most scalable topology under transmission with the less loss rate packets. Topology consideration is the key factor to find out the path among congestion area. So it is important to find out the nodes related to each other's path to reach the desired destination. No cross over is acceptable over path determination policy.

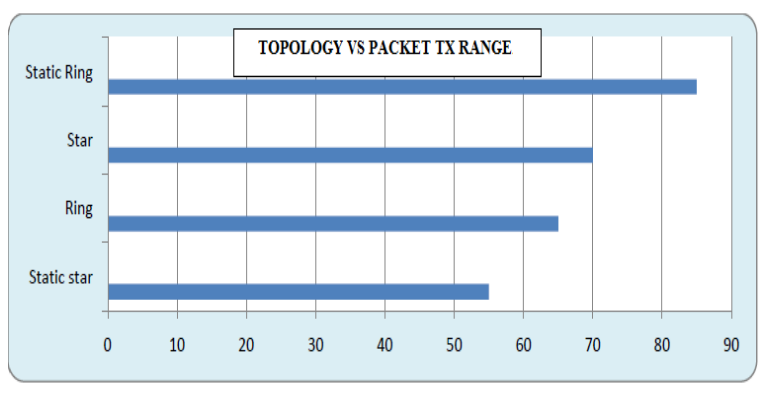

Fig.5 Topological Comparison

Fig. 6 shows the comparison among algorithms GPSR and Greedy in terms of percentage of packets send with respect to the number of nodes. The GPSR is based on adaptive scalable topology mechanism. So its rate of transmission of data packets is efficient than other Greedy algorithms. The packet's drop rate is also minimal in case of GPSR algorithm. So the effective optimization of routing protocol through dynamic topology based on application aspect is adopted.

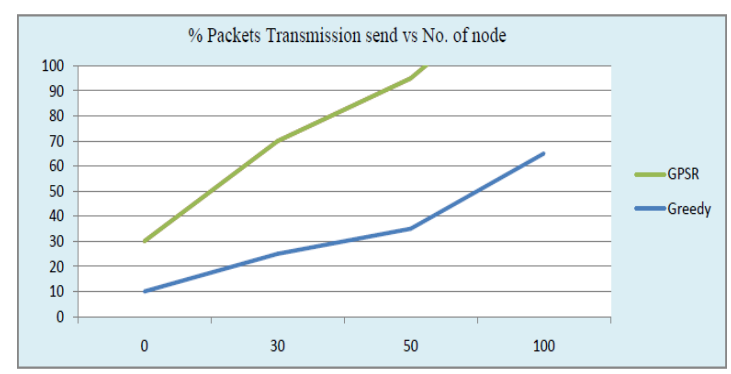

Fig.6. Comparison among GPSR and Greedy

\section{CONCLUSION}

This paper presented Scalable Greedy Perimeter Stateless Routing (SGPSR), a routing algorithm that uses scalability through topology based on traffic demand. SGPSR efficiently delivers $97 \%$ of data packets successfully. It is in competition with other Greedy algorithms like DSR, TORA, AODV including all pause timings with different topological configurations, and give good results than other Greedy algorithms on comparable parameters like percentage of data packets sent, scalability. SGPSR gives routing protocol independent of the length of the routes and generates packets with high mobility. Other Greedy algorithms have chances to fail during

caching overloading. To scale the routing through geographic addressing in addition to the scalable topology is the powerful lever to the optimization of routing in GPSR.

\section{FUTURE PROPOSAL}

GPSR protocol itself decouples participation during route finding as a forwarder from participation in the location data base entries. Only nodes that have information about the further traffic destinations need to send location updates. In a dense network, it is easy to configure only a small subset of sensor nodes to take measurements from source to destination, by flooding some few configurable packets through the network. On the other hand, the rest of the network can provide a robust transmit network to reach the destination without generating any information about traffic to and from the location data base due to traffic overhead. So for far end destinations with traffic overhead it is necessary that queries and data update on routing be geographically addressed.

\section{REFERENCES}

[1] "A Survey of Wireless Sensor Network Abstraction for Application Development" International Journal of Distributed Sensor Networks Volume 2012 (2012), Article ID 740268, 12 pages.

[2] M. Abolhasan, T. Wysocki and E. Dutkiewicz, "A review of routing protocols for mobile ad hoc networks," $\mathrm{Ad}$ Hoc Networks 2, pp. 1-22, 2004.

[3] T. A.Wysocki, A. Dadej, and B. J. Wysocki, "Secure routing protocols for mobile ad-hoc wireless networks," in Advanced Wired and Wireless Networks, Eds. Springer, 2004.

[4] T. Clausen, P. Jacquet, A. Laouiti, P. Muhlethaler, A. Qayyum, L. Viennot, "Optimized link state routing protocol for ad hoc networks," in: Proceedings of IEEE INMIC, December 2001, pp. 62-68.

[5] Zaumen, W., and Garcia-luna Aceves, J. "Dynamics of distributed shortest-path routing algorithms". In Proceedings of the SIGCOMM "91 Conference on 
Communications Architectures, Protocols and Applications (Sept. 1991), pp. 31-42.

[6] Johnson, D. B., and Maltz, D. B. "Dynamic source routing in ad hoc wireless networks". In Mobile Computing, T. Imielinski and H. Korth, Eds. Kluwer Academic Publishers, 1996, ch. 5, pp. 153-181.

[7] Perkins, C. "Ad hoc on demand distance vector (AODV) routing".Internet-draft-draft-ietfmanet-aodv-04.txt, Oct. 1999.

[8] Haas, Z., and Pearlman, M. "The performance of query control schemes for the zone routing protocol". In
Proceedings of the SIGCOMM "98 Conference on Communications Architectures, Protocols and Applications (Sept. 1998).

[9] IEEE computer society Lan Man Standards Committee. Wireless LAN Medium Access Control (MAC) and Physical Layer (PHY) Specifications. IEEE Std. 802.111997.

[10] Chin- Yang Tseng, "A specification based intrusion detection system for AODV". Journal on security of Ad hoc and sensor networks, pp. 305-310, March 2003. 